\title{
A Laguerre-based FDTD method for wave propagation in 2-D anisotropic dispersive materials
}

\author{
Shu-Wen Chen ${ }^{1}$, Feng $\mathbf{L u}^{2,3 a)}$, Yan-Tao Duan ${ }^{3}$, \\ $\mathrm{Li}-$ Yuan $\mathrm{Su}^{3}$, and Yao $\mathrm{Ma}^{3}$ \\ ${ }^{1}$ School of Mathematics and Information Technology, \\ Jiangsu Second Normal University, Nanjing 210013, Jiangsu, P. R. China \\ ${ }^{2}$ Jiangsu Regulatory Bureau of Nuclear and Radiation Safety, \\ Nanjing 210019, P. R. China \\ ${ }^{3}$ National Key Laboratory on Electromagnetic Environmental Effects and \\ Electro-optical Engineering, PLA University of Science and Technology, \\ Nanjing 210007, China \\ a)fenglu76@163.com
}

\begin{abstract}
A Laguerre-based finite-difference time-domain (FDTD) method for two dimensional anisotropic dispersive materials is proposed in this paper. Taking advantage of the property of Laguerre polynomials, the $n$th derivative of field components in Laguerre domain is deduced. The four elements of the tensor permittivity $\overline{\bar{\varepsilon}}$ of the 2-D anisotropic dispersive media can be unified into a general form in frequency domain. Then the general relationship between electric fields and electric flux densities can be derived in Laguerre domain. Using spatial interpolation of transition and central difference scheme, the formulations are obtained. Two examples of wave propagation in anisotropic magnetized plasma are used to validate the efficiency of the proposed method.
\end{abstract}

Keywords: Laguerre, FDTD, anisotropic, dispersive

Classification: Electromagnetic theory

\section{References}

[1] K. S. Yee: IEEE Trans. Antennas Propag. 14 (1966) 302. DOI:10.1109/TAP. 1966.1138693

[2] Y. S. Chung, T. K. Sarkar, B. H. Jung and M. Salazar-Palma: IEEE Trans. Microw. Theory Techn. 51 (2003) 697. DOI:10.1109/TMTT.2003.808732

[3] X. L. Xi, Y. Fang, J. F. Liu and Z. B. Zhu: IEICE Electron. Express 12 (2015) 20150191. DOI:10.1587/elex.12.20150191

[4] W. Shao, B. Z. Wang and X. F. Liu: IEEE Microw. Wireless Compon. Lett. 16 (2006) 308.

[5] P. P. Ding, G. F. Wang, H. Lin and B. Z. Wang: IEEE Microw. Wireless Compon. Lett. 16 (2006) 161. 
[6] T. Namiki: IEEE Trans. Microw. Theory Tech. 47 (1999) 2003. DOI:10.1109/ 22.795075

[7] J. Shibayama, A. Yokomizo, J. Yamauchi and H. Nakano: IEICE Electron. Express 9 (2012) 365. DOI:10.1587/elex.9.365

[8] I. Ahmed, E. K. Chua, E. P. Li and Z. Chen: IEEE Trans. Antennas Propag. 56 (2008) 3596. DOI:10.1109/TAP.2008.2005544

[9] J. Shibayama, A. Ryoji, J. Yamauchi and H. Nakano: IEEE Microw. Wireless Compon. Lett. 8 (2009) 890. DOI:10.1109/LAWP.2009.2028448

[10] R. Siushansian and J. Lovetri: IEEE Microw. Guided Wave Lett. 5 (1995) 426. DOI: $10.1109 / 75.481849$

[11] R. Luebbers, D. Steich and K. Kunz: IEEE Trans. Antennas Propag. 41 (1993) 1249. DOI: $10.1109 / 8.247751$

[12] M. Ha and M. Swaminathan: IEEE Microw. Wireless Compon. Lett. 21 (2011) 225. DOI:10.1109/LMWC.2011.2119296

[13] B. H. Jung, Z. Mei, T. K. Sarkar and M. Salazar-Palma: Microw. Opt. Technol. Lett. 54 (2012) 925. DOI:10.1002/mop.26696

[14] W. J. Chen, W. Shao and B. Z. Wang: IEEE Microw. Wireless Compon. Lett. 23 (2013) 228. DOI:10.1109/LMWC.2013.2253310

[15] D. M. Pozar: Microwave Engineering (Wiley, New York, 2011).

[16] Y. T. Duan, B. Chen, H. L. Chen and Y. Yi: Electron. Lett. 46 (2010) 318. DOI:10.1049/el.2010.3135

[17] S. Liu and S. Q. Liu: Int. J. RF Microwave Comput-Aided Eng. 20 (2010) 465. DOI:10.1002/mmce.20452

[18] H. W. Yang, R. S. Chen and Y. C. Zhou: Int. J. Infrared Milli. Waves 28 (2007) 751.

[19] R. J. Luebbers, F. Hunsberger and K. S. Kunz: IEEE Trans. Antennas Propag. 39 (1991) 29. DOI:10.1109/8.64431

[20] R. J. Luebbers and F. Hunsberger: IEEE Trans. Antennas Propag. 40 (1992) 1297. DOI: $10.1109 / 8.202707$

[21] V. L. Ginzburg: The Propagation of Electromagnetic Waves in Plasma (Pergamon, New York, 1970).

[22] S. Liu, S. Zhong and S. Liu: J. Syst. Eng. Electron. 17 (2006) 290. DOI: 10.1016/S1004-4132(06)60050-9

\section{Introduction}

The conventional finite-difference time-domain (FDTD) method [1] has been proven to be an effective technique for the solution of numerous electromagnetic problems. However, it is difficult to analyze multiscale structures with fine structures such as thin slot, thin layer media and so on because of the CourantFriedrich-Levy (CFL) stability condition. To overcome this limitation, an unconditionally stable FDTD method has been proposed using weighted Laguerre polynomials $[2,3,4,5]$. Different from the conventional FDTD method, the spatial and temporal variables can be separated in the Laguerre-based FDTD method. The Laguerre-based FDTD method is a marching-on-in-order method and is more efficient than the conventional FDTD method. Moreover, this method avoids the numerical dispersion error, which is enlarging with the increase of the time-step size when the ADI-FDTD $[6,7]$ and LOD-FDTD $[8,9]$ are used.

The conventional frequency-dependent FDTD formulation $[10,11]$ has been widely used for dispersive materials such as plasmas, ferrite material, water, and so 
on. And it has been proved to be robust and efficient. Recently, Ha et al. introduced a Laguerre frequency-dependent FDTD formulation for isotropic dispersive materials [12]. Using the time domain formulation of the electric susceptibility for isotropic dispersive media, this method needs to perform the Laguerre transform of the convolution operator. Using the property of convolution, Jung et al. fit the fields, the flux densities, the permittivity and permeability of the medium with a finite sum of orthonormal associated Laguerre basis functions in [13]. And this method is used to analyze transient wave propagation in an arbitrary isotropic dispersive media. Chen et al. introduced the auxiliary differential equation (ADE) Laguerre-based FDTD method to simulate electromagnetic wave propagation in a variety of isotropic dispersive media [14].

The relative electric permittivity of 2-D anisotropic dispersive media is a tensor permittivity $\overline{\bar{\varepsilon}}$ [15], where $\overline{\bar{\varepsilon}}$ is a $2 \times 2$ matrix. The four elements of $\overline{\bar{\varepsilon}}$ are frequencydependent. If the above methods $[12,13,14]$ are used, the deduced formulations will be complicated. So far, only the first and second derivative of field components in Laguerre domain is deduced $[2,12]$. Using the first and second derivative of field components in Laguerre domain, only 2-D formulations for anisotropic-medium PML were derived [16]. The frequency-dependent formulations of anisotropicmedium PML contain $(j \omega)^{n}(n \geq 3)$, and [16] is helpless when anisotropic dispersive materials are involved in computational domain.

In this paper, efficient formulations are proposed to apply the Laguerre-based FDTD method for transient wave propagation in 2-D anisotropic dispersive media. In order to establish the relationship between electric fields and electric flux densities in Laguerre domain, the $n$th derivative of field components in Laguerre domain is deduced. Each element of the tensor permittivity $\overline{\bar{\varepsilon}}$ of anisotropic dispersive media can be unified into the general form. And the relationship between electric fields and electric flux densities is derived in frequency domain. Then transforming frequency domain into time domain and using the $n$th derivative, the relationship between electric fields and electric flux densities is derived in Laguerre domain. Some electric fields are not available directly from the FDTD grid at this moment, which must be interpolated from neighboring quantities [17]. Using central difference scheme, the Laguerre-based FDTD formulations of 2-D anisotropic dispersive media are deduced. To validate the efficiency of the proposed method, two numerical examples are simulated. The efficiency of the proposed method for 2-D anisotropic dispersive media simulation is verified from comparison of the simulated results from the proposed method and that from the SO-FDTD method [18], the RC-FDTD method [19, 20], the TRC-FDTD method [10, 17] and theoretical method [21].

\section{Formulations}

When anisotropic dispersive media is involved, both electric permittivity $\varepsilon$ and magnetic permeability $\mu$ are tensors, need to be transformed into the Laguerre domain. Since magnetic permeability $\mu$ is similar to electric permittivity $\varepsilon$, only electric permittivity is discussed in this paper. 


\subsection{The $n$th derivative of field components in Laguerre domain}

An orthonormal set of basis functions $\left\{\varphi_{0}, \varphi_{1}, \varphi_{2}, \varphi_{3} \ldots\right\}$ can be defined as $\varphi_{p}(\bar{t})=e^{-\bar{t} / 2} L_{p}(\bar{t})$. Here $\bar{t}=s t$ and $s$ is a positive time-scaling factor. $L_{p}(\bar{t})$ is the Laguerre polynomial of order $p$, and $L_{p}(t)$ is defined by $L_{p}(t)=\frac{e^{t}}{p !} \frac{d^{p}}{d t^{p}}\left(t^{p} e^{-t}\right)$ ( $p \geq 0 ; t \geq 0$ ). It is noted that these functions are convergent to zero as $t \rightarrow \infty$. Hence arbitrary functions spanned by these basis functions are also absolutely convergent to zero as $t \rightarrow \infty$.

The first derivative of field components $U(r, t)$ with respect to $t$ is [2]

$$
\frac{\partial U(r, t)}{\partial t}=s \sum_{p=0}^{\infty}\left(0.5 U_{p}(r)+\sum_{k=0, p>0}^{p-1} U_{k}(r)\right) \varphi_{p}(s t)
$$

where $U_{p}(r)$ and $U_{k}(r)$ are the $p$ th and $k$ th Laguerre basis coefficients.

The second derivative can be written as [12]:

$$
\frac{\partial^{2} U(r, t)}{\partial t^{2}}=s^{2} \sum_{p=0}^{\infty}\left(0.25 U_{p}(r)+\sum_{k=0, p>0}^{p-1}(p-k) U_{k}(r)\right) \varphi_{p}(s t)
$$

The $n$th derivative of field components is deduced and can be written as:

$$
\frac{\partial^{n} U(r, t)}{\partial t^{n}}=s^{n} \sum_{p=0}^{\infty}\left(0.5^{n} U_{p}(r)+\sum_{k=0, p>0}^{p-1} W_{n, k}^{p} U_{k}(r)\right) \varphi_{p}(s t)
$$

where $W_{n, k}^{p}$ is satisfied with the analogical relation, $W_{n, k}^{p}=W_{n, k-1}^{p-1}(k \geq 1, p \geq 2)$. If $W_{n, 0}^{p}$ has been derived, $W_{n, k}^{p}$ can also be obtained. Here

$$
W_{n, 0}^{p}=0.5 W_{n-1,0}^{p}+\sum_{k=1}^{p-1} W_{n-1,0}^{k}+0.5^{n-1}(n \geq 1, p \geq 1)
$$

In fact, equation (1) and (2) can be regarded as special case of (3). So they can also be written as the form of equation (3).

\subsection{The relationship between electric fields and electric flux densities in Laguerre domain}

In anisotropic dispersive media, the electric flux densities $D$ are related to the electric field $E$ through the tensor permittivity $\overline{\bar{\varepsilon}}$ of the local tissue by the following equation [15]:

$$
D(\omega)=\varepsilon_{0} \overline{\bar{\varepsilon}}_{r}(\omega) E(\omega)
$$

where

$$
\overline{\bar{\varepsilon}}_{r}(\omega)=\left[\begin{array}{ll}
\varepsilon_{x x}(\omega) & \varepsilon_{x y}(\omega) \\
\varepsilon_{y x}(\omega) & \varepsilon_{y y}(\omega)
\end{array}\right]
$$

And the four elements of the tensor permittivity $\overline{\bar{\varepsilon}}$ can be unified into the general form as: 


$$
\varepsilon_{d i s}(\omega)=\frac{\sum_{n=0}^{N 2} p_{n}(j \omega)^{n}}{\sum_{m=0}^{N 1} q_{m}(j \omega)^{m}}
$$

where $\varepsilon_{d i s}(\omega)$ represents an element in four elements, $\omega$ represents angular frequency, and $p_{n}, q_{m}$ are known constants. According to (5), using the mathematical conversion, we have

$$
\begin{aligned}
& \sum_{m=0}^{R 1} q_{m x}(j \omega)^{m} D_{x}(\omega)=\varepsilon_{0}\left(\sum_{n=0}^{R 2} p_{n x}(j \omega)^{n} E_{x}(\omega)+\sum_{n=0}^{R 3} p_{n y}(j \omega)^{n} E_{y}(\omega)\right) \\
& \sum_{m=0}^{T 1} q_{m y}(j \omega)^{m} D_{y}(\omega)=\varepsilon_{0}\left(\sum_{n=0}^{T 2} p_{n x}(j \omega)^{n} E_{x}(\omega)+\sum_{n=0}^{T 3} p_{n y}(j \omega)^{n} E_{y}(\omega)\right)
\end{aligned}
$$

where $R_{1}, R_{2}$, and $R_{3}$ represent the maximum derivative of $D_{x}(\omega), E_{x}(\omega)$, and $E_{y}(\omega)$ in frequency domain after conversion. And $q_{m x}, p_{n x}$, and $p_{n y}$ in (7) are known constants after mathematical conversion, which can be obtained from $\varepsilon_{x x}(\omega)$ and $\varepsilon_{x y}(\omega)$. The definition of $T_{i}$ is similar to $R_{i}(i=1,2,3)$. The $q_{m y}, p_{n x}$, and $p_{n y}$ in (8) are similar to $q_{m x}, p_{n x}$, and $p_{n y}$ in (7).

Using Laguerre basis functions, the electric field $E$ and electric flux densities $D$ can be represented in Laguerre domain as a sum of Laguerre basis functions scaled by Laguerre basis coefficients and as follows:

$$
\begin{array}{ll}
E_{i}(r, t) & =\sum_{p=0}^{\infty} E_{i}^{p}(r) \varphi_{p}(s t) \quad(i=x, y) \\
D_{i}(r, t) & =\sum_{p=0}^{\infty} D_{i}^{p}(r) \varphi_{p}(s t) \quad(i=x, y)
\end{array}
$$

With the transition relationship from frequency domain to time domain $(j \omega \rightarrow \partial / \partial t),(7)-(8)$ can be converted to time domain. Then inserting (3) into it, we have

$$
\begin{aligned}
& \sum_{p=0}^{\infty} D_{x}^{p}(r) \varphi_{p}(s t)=\sum_{p=0}^{\infty}\left(\varepsilon_{0}\left(\sum_{n=0}^{R_{2}} p_{n x} s^{n} 0.5^{n} E_{x}^{p}(r)+\sum_{n=1}^{R_{2}} p_{n x} s^{n} \sum_{k=0, p>0}^{p-1} W_{n, k}^{p} E_{x}^{k}(r)\right) \varphi_{p}(s t)\right. \\
& \quad+\varepsilon_{0}\left(\sum_{n=0}^{R_{3}} p_{n y} s^{n} 0.5^{n} E_{y}^{p}(r)+\sum_{n=1}^{R_{3}} p_{n y} s^{n} \sum_{k=0, p>0}^{p-1} W_{n, k}^{p} E_{y}^{k}(r)\right) \varphi_{p}(s t) \\
& \left.\quad-\sum_{m=1}^{R_{1}} q_{m x} s^{m} \sum_{k=0, p>0}^{p-1} W_{n, k}^{p} D_{x}^{k}(r) \varphi_{p}(s t)\right) / \sum_{m=0}^{R_{1}} q_{m x} s^{m} 0.5^{m}
\end{aligned}
$$




$$
\begin{aligned}
& \sum_{p=0}^{\infty} D_{y}^{p}(r) \varphi_{p}(s t)=\sum_{p=0}^{\infty}\left(\varepsilon_{0}\left(\sum_{n=0}^{T_{2}} p_{n x} s^{n} 0.5^{n} E_{x}^{p}(r)+\sum_{n=1}^{T_{2}} p_{n x} s^{n} \sum_{k=0, p>0}^{p-1} W_{n, k}^{p} E_{x}^{k}(r)\right) \varphi_{p}(s t)\right. \\
& \quad+\varepsilon_{0}\left(\sum_{n=0}^{T_{3}} p_{n y} s^{n} 0.5^{n} E_{y}^{p}(r)+\sum_{n=1}^{T_{3}} p_{n y} s^{n} \sum_{k=0, p>0}^{p-1} W_{n, k}^{p} E_{y}^{k}(r)\right) \varphi_{p}(s t) \\
& \left.\quad-\left.\sum_{m=1}^{T_{1}} q_{m y} s^{m} \sum_{k=0, p>0}^{p-1} W_{n, k}^{p} D_{k}(r)\right|_{y} \varphi_{p}(s t)\right) / \sum_{m=0}^{T_{1}} q_{m y} s^{m} 0.5^{m}
\end{aligned}
$$

\subsection{Spatial interpolation of transition}

The spatial discretization is performed on a space node. Electric flux density $D_{x}(i, j)$ is located at space node $(i+0.5, j)$. When the relationship between $D_{x}(i, j)$ and $E_{x}(i, j), E_{y}(i, j)$ is established in Laguerre domain, we need to know $E_{x}, E_{y}$ located at space node $(i+0.5, j)$. But in fact $E_{y}(i, j)$ is located at space node $(i, j+0.5)$. Here the spatial interpolation of field adjacent nodes [17] is adopted:

$$
\left.E_{y}\right|_{i+0.5, j}=0.25\left(\left.E_{y}\right|_{i, j+0.5}+\left.E_{y}\right|_{i+1, j+0.5}+\left.E_{y}\right|_{i, j-0.5}+\left.E_{y}\right|_{i+1, j-0.5}\right)
$$

Similarly, $D_{y}(i, j)$ requires the use of $\left.E_{x}\right|_{i, j+0.5}$, and $\left.E_{y}\right|_{i, j+0.5}$. Using the same method, we have

$$
\left.E_{x}\right|_{i, j+0.5}=0.25\left(\left.E_{x}\right|_{i+0.5, j}+\left.E_{x}\right|_{i-0.5, j}+\left.E_{x}\right|_{i+0.5, j+1}+\left.E_{x}\right|_{i-0.5, j+1}\right)
$$

\subsection{Mathematical formulation of Laguerre-based FDTD method for anisotropic dispersive media}

The Laguerre-based FDTD formulations of 2-D anisotropic dispersive media are deduced in this section. For simplicity, only the permittivity (electric susceptibility) for 2-D $\mathrm{TE}_{\mathrm{z}}$ case is discussed in this paper. With anisotropic dispersive media, the Maxwell's equations and constitution relation are:

$$
\begin{aligned}
& \frac{\partial D_{x}}{\partial t}=\frac{\partial H_{z}}{\partial y}-\frac{\partial H_{y}}{\partial z}-J_{x} \\
& \frac{\partial D_{y}}{\partial t}=\frac{\partial H_{x}}{\partial z}-\frac{\partial H_{z}}{\partial x}-J_{y} \\
& \mu_{0} \frac{\partial H_{z}}{\partial t}=\frac{\partial E_{x}}{\partial y}-\frac{\partial E_{y}}{\partial x} \\
& D(\omega)=\varepsilon_{0} \overline{\bar{\varepsilon}}_{r}(\omega) E(\omega)
\end{aligned}
$$

Inserting (3) into (15)-(18), multiplying both sides of the equations by $\varphi_{q}(\bar{t})$ and integrating over $\bar{t}=[0, \infty)$, only the temporal coefficients of order $q$ remain. Substituting electric fields of spatial interpolation, magnetic fields and electric flux densities' temporal coefficients of order $q$ into electric fields, and using central difference scheme, the electric fields of order $q$ are obtained, which are related to magnetic fields and electric flux densities. Then using central difference scheme in magnetic fields and electric flux densities, the following discrete space equations are obtained: 


$$
\begin{aligned}
& -\left.\left.\bar{C}_{y}^{H}\right|_{i, j-1} E_{x}^{q}\right|_{i, j-1}-\left.\left.\bar{C}_{y}^{H}\right|_{i, j} E_{x}^{q}\right|_{i, j+1}+\left.\left(\left.\bar{C}_{y}^{H}\right|_{i, j}+\left.\bar{C}_{y}^{H}\right|_{i, j-1}+\frac{\sum_{n=0}^{R_{2}} p_{n x} s^{n} 0.5^{n}}{\left.\bar{C}_{y}^{E}\right|_{i, j} \cdot \sum_{m=0}^{R_{1}} q_{m x} s^{m} 0.5^{m}}\right) E_{x}^{q}\right|_{i, j} \\
& +\left.\left(\left.\bar{C}_{x}^{H}\right|_{i, j}+\frac{0.25 \sum_{n=0}^{R_{3}} p_{n y} s^{n} 0.5^{n}}{\left.\bar{C}_{y}^{E}\right|_{i, j} \cdot \sum_{m=0}^{R_{1}} q_{m x} s^{m} 0.5^{m}}\right) E_{y}^{q}\right|_{i+1, j}-\left.\left(\left.\bar{C}_{x}^{H}\right|_{i, j}-\frac{0.25 \sum_{n=0}^{R_{3}} p_{n y} s^{n} 0.5^{n}}{\left.\bar{C}_{y}^{E}\right|_{i, j} \cdot \sum_{m=0}^{R_{1}} q_{m x} s^{m} 0.5^{m}}\right) E_{y}^{q}\right|_{i, j} \\
& -\left.\left(\left.\bar{C}_{x}^{H}\right|_{i, j-1}-\frac{0.25 \sum_{n=0}^{R_{3}} p_{n y} s^{n} 0.5^{n}}{\left.\bar{C}_{y}^{E}\right|_{i, j} \sum_{m=0}^{R_{1}} q_{m x} s^{m} 0.5^{m}}\right) E_{y}^{q}\right|_{i+1, j-1}+\left.\left(\left.\bar{C}_{x}^{H}\right|_{i, j-1}+\frac{0.25 \sum_{n=0}^{R_{3}} p_{n y} s^{n} 0.5^{n}}{\left.\bar{C}_{y}^{E}\right|_{i, j} \sum_{m=0}^{R_{1}} q_{m x} s^{m} 0.5^{m}}\right) E_{y}^{q}\right|_{i, j-1} \\
& =-\left.\frac{2}{\left.s \varepsilon_{0} \bar{C}_{y}^{E}\right|_{i, j}} J_{x}^{q}\right|_{i, j}-\left.\frac{2}{\left.\varepsilon_{0} \bar{C}_{y}^{E}\right|_{i, j}} \sum_{k=0, q>0}^{q-1} D_{x}^{k}\right|_{i, j}-2\left(\left.\sum_{k=0, q>0}^{q-1} H_{z}^{k}\right|_{i, j}-\left.\sum_{k=0, q>0}^{q-1} H_{z}^{k}\right|_{i, j-1}\right) \\
& \text { - }\left(\left.\sum_{n=1}^{R_{2}} p_{n x} s^{n} \sum_{k=0, p>0}^{p-1} W_{n, k}^{p} E_{x}^{k}\right|_{i, j}-\left.\frac{1}{\varepsilon_{0}} \sum_{m=1}^{R_{1}} q_{m x} s^{m} \sum_{k=0, p>0}^{p-1} W_{n, k}^{p} D_{x}^{k}\right|_{i, j}+\left.0.25 \sum_{n=1}^{R_{3}} p_{n y} s^{n} \sum_{k=0, p>0}^{p-1} W_{n, k}^{p} E_{y}^{k}\right|_{i, j}\right. \\
& +0.25 \sum_{n=1}^{R_{3}} p_{n y} s^{n} \sum_{k=0, p>0}^{p-1} W_{n, k}^{p} E_{y}^{k} l_{i+1, j}+\left.0.25 \sum_{n=1}^{R_{3}} p_{n y} s^{n} \sum_{k=0, p>0}^{p-1} W_{n, k}^{p} E_{y}^{k}\right|_{i, j-1} \\
& \left.+\left.0.25 \sum_{n=1}^{R_{3}} p_{n y} s^{n} \sum_{k=0, p>0}^{p-1} W_{n, k}^{p} E_{y}^{k}\right|_{i+1, j-1}\right) / \sum_{m=0}^{R_{1}} q_{m x} s^{m} 0.5^{m} /\left.\bar{C}_{y}^{E}\right|_{i, j} \\
& -\left.\left.\bar{C}_{x}^{H}\right|_{i-1, j} E_{y}^{q}\right|_{i-1, j}-\left.\left.\bar{C}_{x}^{H}\right|_{i, j} E_{y}^{q}\right|_{i+1, j}+\left.\left(\left.\bar{C}_{x}^{H}\right|_{i, j}+\left.\bar{C}_{x}^{H}\right|_{i-1, j}+\frac{\sum_{n=0}^{T_{3}} p_{n y} s^{n} 0.5^{n}}{\sum_{m=0}^{T_{1}} q_{m y} s^{m} 0.5^{m} /\left.\bar{C}_{x}^{E}\right|_{i, j}}\right) E_{y}^{q}\right|_{i, j} \\
& +\left.\left(\left.\bar{C}_{y}^{H}\right|_{i, j}+\frac{0.25 \sum_{n=0}^{T_{2}} p_{n x} s^{n} 0.5^{n}}{\left.\bar{C}_{x}^{E}\right|_{i, j} \cdot \sum_{m=0}^{T_{1}} q_{m y} s^{m} 0.5^{m}}\right) E_{x}^{q}\right|_{i, j+1}-\left.\left(\left.\bar{C}_{y}^{H}\right|_{i, j}-\frac{0.25 \sum_{n=0}^{T_{2}} p_{n x} s^{n} 0.5^{n}}{\left.\bar{C}_{x}^{E}\right|_{i, j} \cdot \sum_{m=0}^{T_{1}} q_{m y} s^{m} 0.5^{m}}\right) E_{x}^{q}\right|_{i, j} \\
& -\left.\left(\left.\bar{C}_{y}^{H}\right|_{i-1, j}-\frac{0.25 \sum_{n=0}^{T_{2}} p_{n x} s^{n} 0.5^{n}}{\left.\bar{C}_{x}^{E}\right|_{i, j} \sum_{m=0}^{T_{1}} q_{m y} s^{m} 0.5^{m}}\right) E_{x}^{q}\right|_{i-1, j+1}+\left.\left(\left.\bar{C}_{y}^{H}\right|_{i-1, j}+\frac{0.25 \sum_{n=0}^{T_{2}} p_{n x} s^{n} 0.5^{n}}{\left.\bar{C}_{x}^{E}\right|_{i, j} \sum_{m=0}^{T_{1}} q_{m y} s^{m} 0.5^{m}}\right) E_{x}^{q}\right|_{i-1, j} \\
& =-\left.\frac{2}{\left.s \varepsilon_{0} \bar{C}_{x}^{E}\right|_{i, j}} J_{y}^{q}\right|_{i, j}-\left.\frac{2}{\left.\varepsilon_{0} \bar{C}_{x}^{E}\right|_{i, j}} \sum_{k=0, q>0}^{q-1} D_{y}^{k}\right|_{i, j}-2\left(\left.\sum_{k=0, q>0}^{q-1} H_{z}^{k}\right|_{i-1, j}-\left.\sum_{k=0, q>0}^{q-1} H_{z}^{k}\right|_{i, j}\right) \\
& \text { - }\left(\left.\sum_{n=1}^{T_{3}} p_{n y} s^{n} \sum_{k=0, p>0}^{p-1} W_{n, k}^{p} E_{y}^{k}\right|_{i, j}-\left.\frac{1}{\varepsilon_{0}} \sum_{m=1}^{T_{1}} q_{m y} s^{m} \sum_{k=0, p>0}^{p-1} W_{n, k}^{p} D_{y}^{k}\right|_{i, j}+\left.0.25 \sum_{n=1}^{T_{2}} p_{n x} s^{n} \sum_{k=0, p>0}^{p-1} W_{n, k}^{p} E_{x}^{k}\right|_{i, j}\right. \\
& +\left.0.25 \sum_{n=1}^{T_{2}} p_{n x} s^{n} \sum_{k=0, p>0}^{p-1} W_{n, k}^{p} E_{x}^{k}\right|_{i, j+1}+0.25 \sum_{n=1}^{T_{2}} p_{n x} s^{n} \sum_{k=0, p>0}^{p-1} W_{n, k}^{p} E_{x}^{k} l_{i-1, j} \\
& \left.+\left.0.25 \sum_{n=1}^{T_{2}} p_{n x} s^{n} \sum_{k=0, p>0}^{p-1} W_{n, k}^{p} E_{x}^{k}\right|_{i-1, j+1}\right) / \sum_{m=0}^{T_{1}} q_{m y} s^{m} 0.5^{m} /\left.\bar{C}_{x}^{E}\right|_{i, j}
\end{aligned}
$$




$$
\begin{aligned}
& \left.H_{z}^{q}\right|_{i, j}=\left.\bar{C}_{y}^{H}\right|_{i, j}\left(\left.E_{x}^{q}\right|_{i, j+1}-\left.E_{x}^{q}\right|_{i, j}\right)-\left.\bar{C}_{x}^{H}\right|_{i, j, k}\left(\left.E_{y}^{q}\right|_{i+1, j}-\left.E_{y}^{q}\right|_{i, j}\right)-\left.2 \sum_{k=0, q>0}^{q-1} H_{z}^{k}\right|_{i, j} \quad \text { (21) } \\
& \left.D_{x}^{q}\right|_{i, j}=\left(\varepsilon _ { 0 } \left(\left.\sum_{n=0}^{R_{2}} p_{n x} s^{n} 0.5^{n} E_{x}^{q}\right|_{i, j}+\left.\sum_{n=1}^{R_{2}} p_{n x} s^{n} \sum_{k=0, q>0}^{q-1} W_{n, k}^{p} E_{x}^{k}\right|_{i, j}+\left.0.25 \sum_{n=0}^{R_{3}} p_{n y} s^{n} 0.5^{n} E_{y}^{q}\right|_{i, j}\right.\right. \\
& +\left.0.25 \sum_{n=1}^{R_{3}} p_{n y} s^{n} \sum_{k=0, q>0}^{q-1} W_{n, k}^{p} E_{y}^{k}\right|_{i, j}+\left.0.25 \sum_{n=0}^{R_{3}} p_{n y} s^{n} 0.5^{n} E_{y}^{q}\right|_{i+1, j}+\left.0.25 \sum_{n=1}^{R_{3}} p_{n y} s^{n} \sum_{k=0, q>0}^{q-1} W_{n, k}^{p} E_{y}^{k}\right|_{i+1, j} \\
& +\left.0.25 \sum_{n=0}^{R_{3}} p_{n y} s^{n} 0.5^{n} E_{y}^{q}\right|_{i, j-1}+\left.0.25 \sum_{n=1}^{R_{3}} p_{n y} s^{n} \sum_{k=0, q>0}^{q-1} W_{n, k}^{p} E_{y}^{k}\right|_{i, j-1}+\left.0.25 \sum_{n=0}^{R_{3}} p_{n y} s^{n} 0.5^{n} E_{y}^{q}\right|_{i+1, j-1} \\
& \left.\left.+\left.0.25 \sum_{n=1}^{R_{3}} p_{n y} s^{n} \sum_{k=0, q>0}^{q-1} W_{n, k}^{p} E_{y}^{k}\right|_{i+1, j-1}\right)-\left.\sum_{m=1}^{R_{1}} q_{m x} s^{m} \sum_{k=0, q>0}^{q-1} W_{n, k}^{p} D_{x}^{k}\right|_{i, j}\right) / \sum_{m=0}^{R_{1}} q_{m x} s^{m} 0.5^{m} \quad \text { (22) } \\
& \left.D_{y}^{q}\right|_{i, j}=\left(\varepsilon _ { 0 } \left(\left.\sum_{n=0}^{T_{3}} p_{n y} s^{n} 0.5^{n} E_{y}^{q}\right|_{i, j}+\left.\sum_{n=1}^{T_{3}} p_{n y} s^{n} \sum_{k=0, q>0}^{q-1} W_{n, k}^{p} E_{y}^{k}\right|_{i, j}+\left.0.25 \sum_{n=0}^{T_{2}} p_{n x} s^{n} 0.5^{n} E_{x}^{q}\right|_{i, j}\right.\right. \\
& +\left.0.25 \sum_{n=1}^{T_{2}} p_{n x} s^{n} \sum_{k=0, q>0}^{q-1} W_{n, k}^{p} E_{x}^{k}\right|_{i, j}+\left.0.25 \sum_{n=0}^{T_{2}} p_{n x} s^{n} 0.5^{n} E_{x}^{q}\right|_{i, j+1}+\left.0.25 \sum_{n=1}^{T_{2}} p_{n x} s^{n} \sum_{k=0, q>0}^{q-1} W_{n, k}^{p} E_{x}^{k}\right|_{i, j+1} \\
& +\left.0.25 \sum_{n=0}^{T_{2}} p_{n x} s^{n} 0.5^{n} E_{x}^{q}\right|_{i-1, j}+\left.0.25 \sum_{n=1}^{T_{2}} p_{n x} s^{n} \sum_{k=0, q>0}^{q-1} W_{n, k}^{p} E_{x}^{k}\right|_{i-1, j}+\left.0.25 \sum_{n=0}^{T_{2}} p_{n x} s^{n} 0.5^{n} E_{x}^{q}\right|_{i-1, j+1} \\
& \left.\left.+\left.0.25 \sum_{n=1}^{T_{2}} p_{n x} s^{n} \sum_{k=0, q>0}^{q-1} W_{n, k}^{p} E_{x}^{k}\right|_{i-1, j+1}\right)-\left.\sum_{m=1}^{T_{1}} q_{m y} s^{m} \sum_{k=0, q>0}^{q-1} W_{n, k}^{p} D_{y}^{k}\right|_{i, j}\right) / \sum_{m=0}^{T_{1}} q_{m y} s^{m} 0.5^{m} \quad(23)
\end{aligned}
$$

where $\left.\bar{C}_{x}^{E}\right|_{i, j}=\frac{2}{s \varepsilon_{0} \Delta \bar{x}_{i}},\left.\bar{C}_{y}^{E}\right|_{i, j}=\frac{2}{s \varepsilon_{0} \Delta \bar{y}_{j}},\left.\bar{C}_{x}^{H}\right|_{i, j}=\frac{2}{s \mu_{0} \Delta x_{i}},\left.\bar{C}_{y}^{H}\right|_{i, j}=\frac{2}{s \mu_{0} \Delta y_{j}}$. Here $\Delta x_{i}$ and $\Delta y_{j}$ are the lengths of the cell edge where the electric fields are located. $\Delta \bar{x}_{i}$ and $\Delta \bar{y}_{j}$ are the distances between the center nodes where the magnetic fields are located.

It is noted that $(i, j)$ is not a real position but an array index of each field variable in the above difference equations. From (19)-(20), it can also be found that each electric field variable is related to the six adjacent electric fields. Therefore, each row has seven nonzero terms. The magnetic fields and electric flux densities are known because their orders are lower than those of the electric fields. With reference to Chung et al. [2], equations (19)-(20) can be written as a matrix equation form. The electric fields are obtained from solving the matrix equation. In addition, the relationship between magnetic fields and electric fields is performed from (21). The relationship between electric flux densities and electric fields is performed from (22)-(23). Using (21)-(23), (19)-(20) enable recursive calculation of Laguerre coefficients using previous coefficients for electromagnetic waves.

\section{Numerical demonstrations}

In this section, two examples are presented to demonstrate the efficiency of the proposed method. 


\subsection{Example A:}

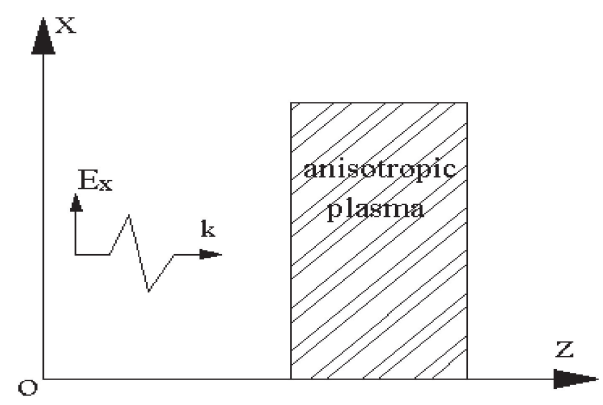

Fig. 1. Calculated geometry.

A one-dimensional plane wave traveling in anisotropic plasma with collisions is discussed here, whose direction is parallel to $z$ axes shown as Fig. 1. The well known tensor permittivity $\overline{\bar{\varepsilon}}$ is expressed as [22]:

$$
\overline{\bar{\varepsilon}}_{r}(\omega)=\left|\begin{array}{cc}
\varepsilon_{x x}(\omega) & j \varepsilon_{x y}(\omega) \\
-j \varepsilon_{y x}(\omega) & \varepsilon_{y y}(\omega)
\end{array}\right|
$$

where

$$
\begin{aligned}
& \varepsilon_{x x}(\omega)=\varepsilon_{y y}(\omega)=1-\frac{\left(\frac{\omega_{p}}{\omega}\right)^{2}\left(1-j \frac{v}{\omega}\right)}{\left(1-j \frac{\nu}{\omega}\right)^{2}-\left(\frac{\omega_{b}}{\omega}\right)^{2}} \\
& j \varepsilon_{x y}(\omega)=j \varepsilon_{y x}(\omega)=\frac{j\left(\frac{\omega_{p}}{\omega}\right)^{2}\left(\frac{\omega_{b}}{\omega}\right)}{\left(1-j \frac{v}{\omega}\right)^{2}-\left(\frac{\omega_{b}}{\omega}\right)^{2}}
\end{aligned}
$$

where $v$ is the electron collision frequency, $\omega_{p}$ is the plasma frequency, and $\omega_{b}$ is the electron cyclotron frequency.

A plane wave traveled in a magnetized collision plasma slab with a thickness of $1.5 \mathrm{~cm}$, and the transmission and reflection coefficients of left-hand circular polarization wave and right-hand circular polarization wave were computed [22]. The Mur's 1st-order absorbing boundary conditions are used to truncate the computational domain. The plasma parameters are $\omega_{\mathrm{p}}=2 \pi \times 28.7 \times 10^{9} \mathrm{rad} / \mathrm{s}$, $\omega_{\mathrm{b}}=1.0 \times 10^{11} \mathrm{rad} / \mathrm{s}$, and $v=20 \mathrm{GHz}$. A Gaussian-derivative pulse is used as an incident electric current profile:

$$
J(t)=\exp \left(-\left(\frac{t-T_{c}}{T_{d}}\right)^{2}\right) \sin \left(2 \pi f_{c}\left(t-T_{c}\right)\right)
$$

where $T_{d}=1 /\left(2 f_{c}\right), T_{c}=3 T_{d}$ and $f_{c}=3 \mathrm{GHz}$. Here, we choose $T_{f}=2 \mathrm{~ns}$, $s=1.8 \times 10^{10}$ and $N L=350$ [2]. The problem is simulated with the proposed method. In order to obtain the good results, we choose the cell size of $0.1 \mathrm{~mm}$, the time step $(\Delta t)$ of $0.167 \mathrm{ps}$. And the transmission and reflection coefficients are calculated with the fast Fourier transform (FFT) technique. Fig. 2 and Fig. 3 
display the magnitudes of the reflection coefficients and transmission coefficients of the left-hand and right-hand circular polarization wave, respectively. To provide a benchmark for comparison, this problem is also solved using theoretical algorithm [21]. As we can see in Fig. 2 and Fig. 3, the results computed from the proposed FDTD method exhibit good agreement with those from the analytical solutions in frequency domain.

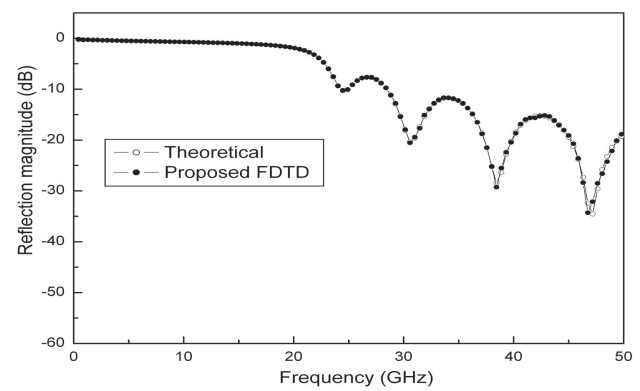

(a)

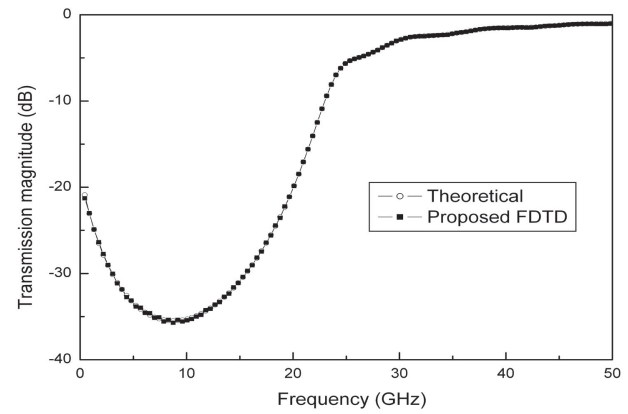

(b)

Fig. 2. Numerical results of left-hand circular polarization wave. (a) Reflection coefficient magnitude versus frequency; (b) transmission coefficient magnitude versus frequency.

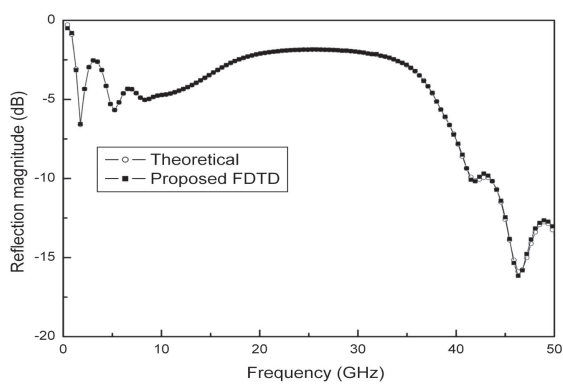

(a)

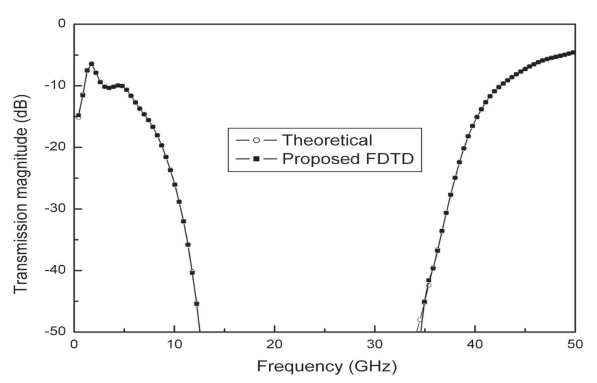

(b)

Fig. 3. Numerical results of right-hand circular polarization wave. (a) Reflection coefficient magnitude versus frequency; (b) transmission coefficient magnitude versus frequency.

\subsection{Example B:}

As shown in Fig. 4, a 2-D parallel plate waveguide with the size of $50 \mathrm{~mm} \times 12 \mathrm{~mm}$ is calculated with the proposed Laguerre-based FDTD method. The parameters of the anisotropic plasma are the same as example A and its thickness is $10 \mathrm{~mm}$. The Mur's 1st-order absorbing boundary conditions are also used to truncate the computational domain. To limit numerical dispersion, a fine grid division with the cell size of $0.1 \mathrm{~mm} \times 0.1 \mathrm{~mm}$ is applied to SO-FDTD, RCFDTD and the TRC-FDTD methods.

The electric field at points P computed by SO-FDTD, RC-FDTD, TRC-FDTD and the proposed methods is monitored and graphed in Fig. 5. It is clear that the calculated field by the proposed method is in good agreement with that by other 
three methods. Table I shows the comparison of the computing time of these methods. It can be seen that the time usage of the proposed method is only about $6.0 \%$ of the time usage of SO-FDTD, about $6.3 \%$ of the time usage of RC-FDTD, and about $6.1 \%$ of the time usage of TRC-FDTD. But the memory requirement of the proposed method is larger than other methods. It can also be seen that the memory requirement of the proposed method is about 15.4 times of the memory requirement of SO-FDTD, about 19.4 times of the memory requirement of RCFDTD, and about 17.0 times of the memory requirement of TRC-FDTD. It should be noted that all calculations in this paper have been performed on a Celeron (R) Dual-Core CPU T3000 1.80 GHz machine with 2G RAM.

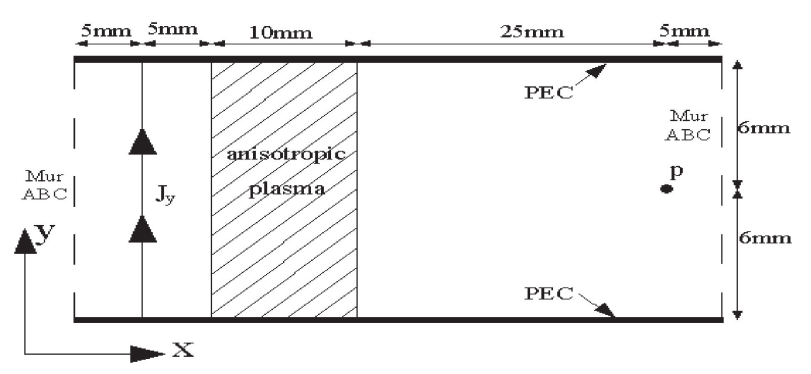

Fig. 4. 2-D parallel plate waveguide with anisotropic plasma.

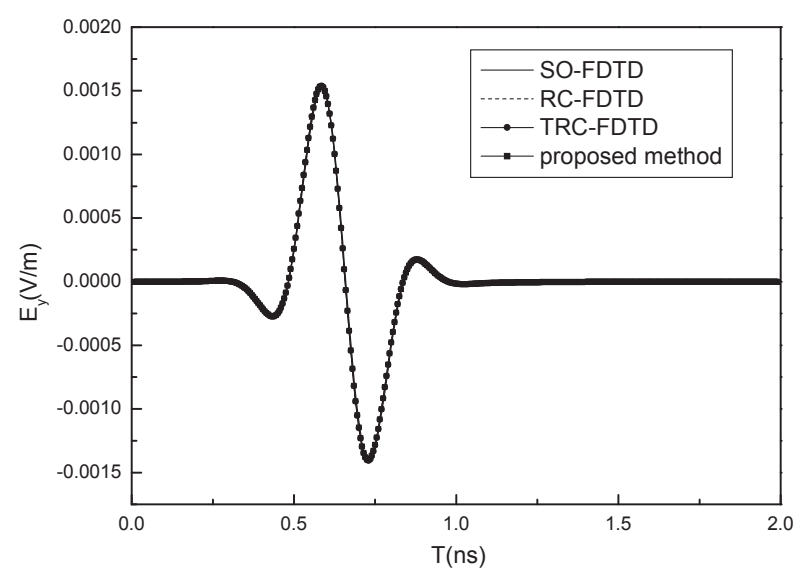

Fig. 5. Electric field calculated from the proposed method and three FDTD methods.

Table I. Comparison of computation time for the 2-D waveguide

\begin{tabular}{|c|c|c|c|c|c|}
\hline Method & $\Delta \mathrm{t}(\mathrm{ps})$ & Meshing size & $\begin{array}{c}\text { Marching } \\
\text { steps }\end{array}$ & Memory & CPU Time (s) \\
\hline SO-FDTD & $\mathbf{0 . 1 6 7}$ & $500 \times 120$ & 12000 & $9.2 \mathrm{M}$ & 290.4 \\
\hline RC-FDTD & $\mathbf{0 . 1 6 7}$ & $500 \times 120$ & 12000 & $7.3 \mathrm{M}$ & $\mathbf{2 7 2 . 8}$ \\
\hline TRC-FDTD & $\mathbf{0 . 1 6 7}$ & $\mathbf{5 0 0} \times 120$ & 12000 & $\mathbf{8 . 3 M}$ & $\mathbf{2 8 3 . 6}$ \\
\hline Proposed method & 1 & $500 \times 120$ & 150 & $\mathbf{1 4 1 . 5 M}$ & 17.3 \\
\hline
\end{tabular}




\section{Conclusions}

The $n$th derivative of field components in Laguerre domain is deduced for wave propagation in anisotropic dispersive materials in this paper. The relationship between electric fields and electric flux densities is established in the Laguerre domain. A new electric field matrix equation for 2-D anisotropic dispersive materials is obtained. Then expansion coefficients of the electromagnetic field components can be derived for each order. Compared with the results of the other methods in two structures, the accuracy of the proposed formulation is verified. It is also demonstrated that the CPU time is greatly saved when the proposed method is used. This method is unconditional stable and the solution is independent of the time discretization, and can be used in multiscale structures with fine structure in anisotropic dispersive materials. 